\title{
Fundamentos jurídicos que favorecen el abordaje de lo rural desde la perspectiva intercultural
}

Por Willam R. Barragán Morocho ${ }^{1}$

\section{RESUMEN}

El siguiente artículo plantea el análisis y reflexión de los lineamientos internacionales sobre las condiciones que permiten un abordaje de lo rural desde una perspectiva intercultural dirigido a la población rural. En un primer momento se hace referencia a la mirada de los organismos internacionales acerca de los derechos de la población rural, en los que se aborda el derecho a la educación, como uno de los derechos fundamentales del hombre que permita mejorar las condiciones de vida y de trabajo de las poblaciones rurales. Posteriormente se plantea el aporte del enfoque intercultural como elemento fundamental que promueve relaciones interpersonales igualitarias, reconociendo las diferencias sociales y culturales de los educandos al interior de los establecimientos educativos como también fuera de ellos, queda cuenta de la educación en contextos educativos rurales.

Palabras clave: organismos internacionales, población rural, intercultural.

\section{Introducción}

Como referente histórico importante de este artículo se encuentra lo rural, constructo que surge en la Edad Media, en la época feudal, posterior a esto surge una nueva clase social denominada burguesía y con ella el surgimiento y la consolidación de las ciudades, donde se desarrollan las actividades políticas y económicas, iniciando así su historia de marginación y subordinación de los sectores rurales, como proveedores de alimentos y materia prima para las ciudades. Apreciándose desde aquella época la existencia de dos clases sociales bien diferenciadas, una clase dominante y otra clase dominada, siendo las primeras que imponen sus conocimientos y la segunda la receptora de sus conocimientos, que ha sido una constante que prevalece hasta nuestros días.

Con el surgimiento del Estado, persisten las clases sociales, sin embargo, las personas toman la denominación de ciudadanos, como sujetos de derechos y obligaciones, entre los derechos más importantes está el derecho a la educación. Consientes que la educación es una herramienta que propicia la libertad y el desarrollo integral de las personas.

1 DrC. Willam R. Barragán Morocho. Universidad de Santiago de Chile. Facultad de Humanidades. Programa Doctorado en Ciencias de la Educación con mención en Educación Intercultural. Email: wbarragam@yahoo.es 
No obstante la educación ha sido utilizada como un instrumento de dominación, a través de la cual grupos de poder imponían sus conocimientos mediante un currículo monocultural que homogeniza a la población, invisibilizando a los diferentes, a los "otros", a los más débiles.

Sin embargo, los avances tecnológicos surgidos por la globalización, ha propiciado que los sectores rurales adquieran un mayor conocimiento de diferentes realidades y en diferentes ámbitos, que despierta un mayor interés de superación y una visión más ambiciosa por alcanzar nuevos objetivos que les permita ser visibilizados e integrados en las sociedades globales como ciudadanos del mundo.

Dado que "la educación rural (...) es diferenciada en los sectores sociales, que a medida que esta se acerca a los sectores más pobres la calidad de la educación va disminuyendo sustancialmente, por consiguiente existe una desigualdad en la distribución del conocimiento, consecuencia de esto es imposible la igualdad de oportunidades, requisito mínimo para la existencia de la democracia".(FAO - UNESCO, 2004, p.124).

Estas inequidades existentes en la ruralidad, demanda una mejor calidad de educación en las escuelas rurales, acordes a las realidades socioculturales de la niñez y la adolescencia, de cada sector, que valore y reconozca su diversidad social y cultural, que integre los elementos que confluyen la labor educativa (alumnos, profesores, apoderados y comunidad), para mejorar los procesos de enseñanza aprendizaje, que contribuya a mejorar la calidad de vida de sus habitantes.

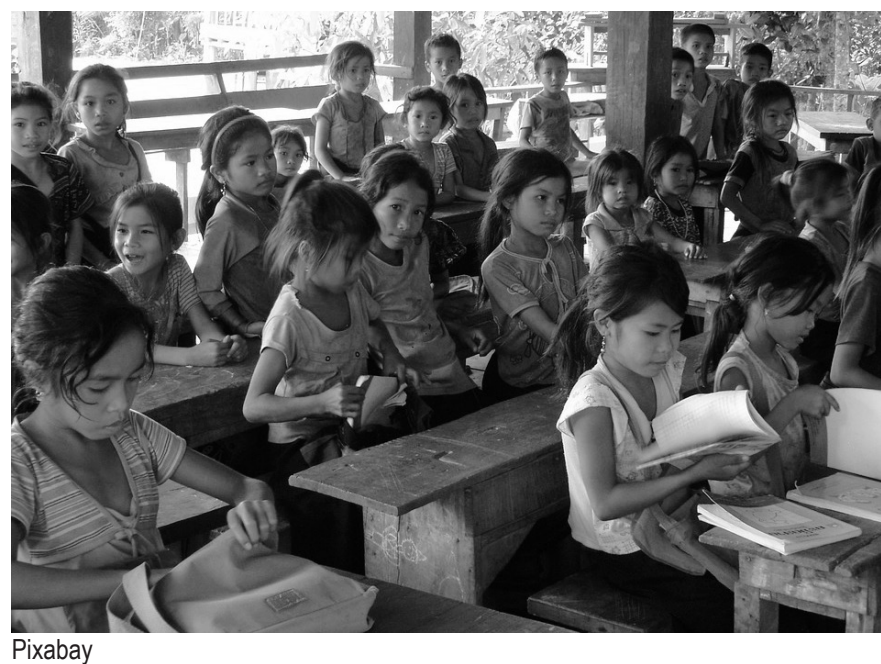

En este contexto surge la educación intercultural, que se orienta por las directrices de los diferentes organismos internacionales de cooperación que resguardan los derechos humanos de la diversidad cultural, visibilizando los derechos de las poblaciones rurales. La educación intercultural desde estas normativas tiende a la integración, conocimiento y entendimiento, permeabilizando esas barreras dicotómicas o constructos del "otro", diferente segregado invisibilizado, al tiempo que mejore la aceptación, valoración y reconocimiento de la diversidad cultural que son tan particulares y diversas a la vez, "para lograr la igualdad de oportunidades (entendida como oportunidades de elección y de acceso) a recursos sociales, económicos y educativos, la superación del racismo y la competencia intercultural en todas las personas, sea cual sea su grupo cultural de referencia" (Aguado, 2005).

Siendo esta la realidad como se ha venido dando, las directrices de los organismos internacionales, creando jurisprudencia en el ámbito internacional que oriente y guíe a los Estadosy organismos nacionales en favor de los derechos de la diversidad cultural, el objetivo de este artículo está centrado en el rol que cumplen los organismos internacionales (ONU y la UNESCO), a través de los convenios y tratados internacionales en favor de los derechos humanos y de la educación intercultural, en favor de una educación más inclusiva y diversa, que reconozca la diversidad cultural.

\section{Ruralidad e interculturalidad}

Conocer y ubicar cuando y donde situar en la historia la génesis de la garantía de los derechos de la diversidad cultural, implica acudir y enfocarse en la Declaración de los Derechos Humanos como fuente inspiradora de lo que se ha denominado la plataforma de los instrumentos jurídicos base de derechos humanos en la historia. Desde la aprobación y vigencia de la Declaración Universal de los Derechos Humanos, estos derechos constituyen un hito civilizatorio y cultural en todas las sociedades:

Su universalidad está dada, al menos, en torno a tres dimensiones: una racional, que los adscribe a todos los seres humanos; una temporal, que los hace válidos en cualquier momento de la historia; $\mathrm{y}$ una espacial, que refiere la extensión de una cultura 
basada en los derechos humanos a todos los países y comunidades. (INDH, 2010).

Considerando, las dimensiones antes mencionadas, estos derechos se constituyen en un instrumento indispensable utilizado por la sociedad y por ende por los Estados, mediante el cual los individuos pueden desarrollar de manera íntegra sus capacidades, para relacionarse con los demás. Estos elementos son vinculantes para preservar y garantizar el desarrollo cultural, permite reproducir y mantener las costumbres, tradiciones y formas de vida de una sociedad o Estado determinado respetando la diversidad social y cultural.

Desde el surgimiento de la Declaración Universal de los Derechos Humanos llevando en vigencia un poco más de medio siglo, plantea que el Estados como institución política legalmente constituida, que direcciona el orden jurídico en sus territorios, garantiza y facilita la aplicación efectiva de estos derechos aceptados universalmente, "tales derechos esenciales a la naturaleza

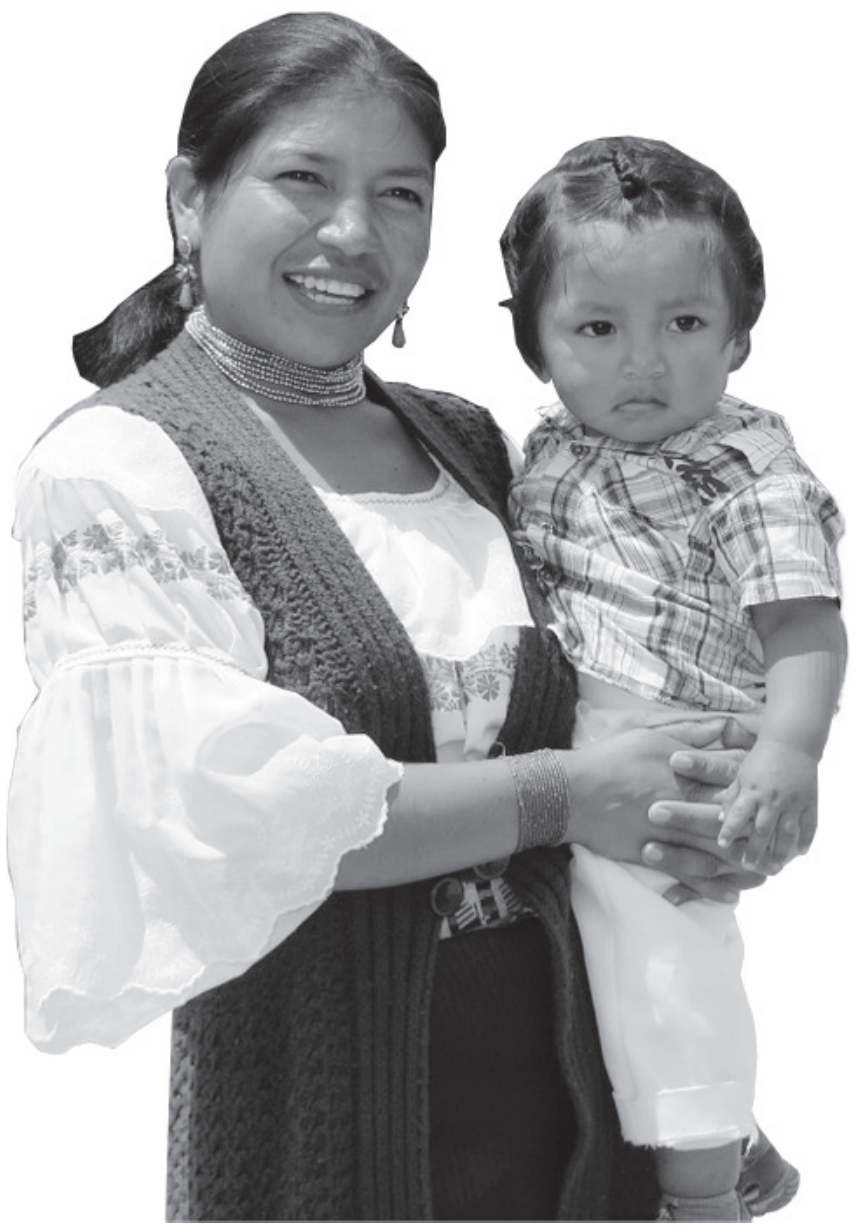

del ser humano, son parte fundamental de toda teoría y práctica democrática, (...) a través del cual se mide el grado de desarrollo de las sociedades y el compromiso de los Estados"(INDH, 2010, p. 9).

Por lo tanto:

Los Estados tienen la obligación de promover, respetar y garantizar los derechos humanos sin discriminación a todas las personas que viven en una misma comunidad. Proceso que se inició en América Latina a finales de los años 80, producto de las sucesivas reformas constitucionales que incorporaron en sus textos un enfoque universal de tales derechos (INDH, 2010, p.10).

En el marco de derechos y libertades fundamentales inherentes a las personas y legalmente aceptadas y reconocidos por los Estado partes, la población rural ha sido uno de los grupos minoritarios más vulnerados en sus derechos humanos universalmente aceptados, sin embargo, la población rural tiene referente internacionales como la ONU y la UNESCO que resguardan y promueven sus derechos legalmente constituidos.

Siendo la educación el instrumento más importante para el desarrollo íntegro de las personas, la UNESCO en la convención relativa a la lucha contra las discriminaciones en la esfera de la enseñanza, en 1960, se propone instituir la cooperación entre las naciones a fin de asegurar el respeto universal de derechos humanos y una igualdad de posibilidades de educación.

En el Artículo 4 señala que:

Los Estados Partes en la presente Convención se comprometen, además a formular, desarrollar y aplicar una política nacional encaminada a promover, por métodos adecuados a las circunstancias y las prácticas nacionales, la igualdad de posibilidades y de trato en la esfera de la enseñanza y, en especial a:

a. Hacer obligatoria y gratuita la enseñanza primaria, generalizar y hacer accesible a toda la enseñanza secundaria en sus diversas formas; hacer accesible a todos, en condiciones de igualdad total y según la capacidad de cada uno, la enseñanza superior; 
velar por el cumplimiento por todos de la obligación escolar prescrita por la ley.

b. Mantener en todos los establecimientos públicos del mismo grado una enseñanza del mismo nivel y condiciones equivalentes en cuanto se refiere a la calidad de la enseñanza proporcionada.

c. Siguiendo la misma línea, la Organización de las Naciones Unidas para la Educación la Ciencia y la Cultura (UNESCO) en las actas de la Conferencia General realizada en París (1974). Su objetivo es proseguir y consolidar un programa a largo plazo de enseñanza y formación en favor de las zonas rurales y en particular a:

- Fomentar, en la perspectiva de la educación permanente y mediante la realización de estudios y experiencias piloto, la adopción de medidas encaminadas a aumentar las oportunidades de acceso a la educación de la población rural y a ofrecerle medios de educación escolar y extraescolar que respondan a las exigencias de su promoción profesional, social y cultural;

- Contribuir al desarrollo de la enseñanza agrícola mediante la formación en todos los niveles de personal científico y técnico adecuado, en particular fortaleciendo y ampliando la formación de maestros, animadores e instructores para las regiones rurales y continuando con ese fin la organización de cursos de perfeccionamiento, internacionales y regionales, de seminarios y de simposios.

Las políticas educativas han sido uno de los derechos reivindicativos en favor de las poblaciones rurales, las cuales han sido insuficientes en la eliminación de las desigualdades en el campo educativo, por lo cual la UNESCO en las actas de la conferencia general realizada en Sofía (1985) en la sección referente "la educación para todos" señala que el derecho a la educación constituye uno de los derechos fundamentales del hombre y es una de las condiciones de la realización de sus demás derechos y que el ejercicio de este derecho aún está lejos de ser efectivo en todo el mundo y que el analfabetismo sigue siendo uno de los grandes males sociales contemporáneos y un gran desafío para la comunidad internacional (p. 23).
En esta conferencia la UNESCO también aprobó el programa "Desarrollo de la educación en las zonas rurales". Su finalidad es el mejoramiento de la educación en las zonas rurales para aplicar estrategias y promover medidas legislativas, administrativas, educativas y financieras destinadas a:

- Reducir las disparidades entre los habitantes del campo y las ciudades, y asegurarles a todos la igualdad de oportunidades y de trato educativos;

- mejorar la calidad y la pertinencia de la educación en las zonas rurales mediante el fortalecimiento de los vínculos entre la educación y el trabajo productivo, la enseñanza de tecnologías destinadas a lograr el desarrollo de las zonas rurales, y la formación de un personal docente que ejerza en el medio rural;

- promover la participación de la población rural en la elaboración y la aplicación de medidas favorables al desarrollo y el mejoramiento de la educación;

- aumentar la contribución de la educación general (...) la modernización de las zonas rurales, así como al mejoramiento de las condiciones de vida y de trabajo de las poblaciones rurales, (...) mediante un mejoramiento de la calidad de la enseñanza agrícola que le permita responder a las exigencias científicas, técnicas y sociales del desarrollo de las zonas rurales (UNESCO, 1985, p.. 25-26).

Los lineamientos desarrollados por la UNESCO se focalizan en promover una educación vinculada con labores agrícolas y productivas, es decir una educación pertinente que respondan a las realidades culturales de sus habitantes, mediante la cual logran mejorar las condiciones de vida.

En esta línea La Conferencia Mundial de Educación para Todos (EPT), realizada en Jomtien, Tailandia, (1990), promueve el derecho a la educación, como elemento trascendental que facilite alcanzar la igualdad entre la diversidad multicultural, como se detalla a continuación: 
Artículo 4.-

Universalizar el acceso a la educación y fomentar la equidad Literal (2) Hay que empeñarse activamente en modificar las desigualdades en materia de educación y suprimir las discriminaciones en las posibilidades de aprendizaje de los grupos desasistidos: los pobres, los niños de la calle y los niños que trabajan, las poblaciones de las zonas remotas y rurales, (p.10).

La persistencia de desigualdades en los sectores rurales respecto del derecho a la educación, factor que influye directamente en la persistencia del analfabetismo y la pobreza rural que impide el desarrollo económico, social y cultural de las poblaciones rurales. Razones más que suficientes por las cuales el foco de atención de la FAO y la UNESCO se centra en la reducción de la pobreza, la seguridad alimentaria y la educación básica como el núcleo del nuevo discurso sobre la asistencia al desarrollo. Sin embargo, a menudo se descuida la índole rural de estos retos. La gran mayoría de la población rural pobre depende de la agricultura para su supervivencia. Por consiguiente, el desarrollo rural afronta un desafío clave para lograr tanto la reducción de la pobreza como la educación para todos (FAO-UNESCO, 2004, p.22).

Como es posible observar, los organismos internacionales de cooperación otorgan los lineamientos a los organismos nacionales, orientados al reconocimiento y respeto de los derechos de la diversidad social y cultural, que se focaliza en el resguardo de los Derechos Humanos que busca la equidad, la justicia y la paz social para construir un mundo donde quepan todos los mundos.

\section{Directrices internacionales acerca de Educación Intercultural}

En el mundo actual donde lo cotidiano es observar cada vez sociedades más diversas, resulta indispensable promover una interacción más armoniosa, justa y equitativa entre la diversidad social, respetando sus identidades culturales, estas realidades cada día más generalizadas permiten crear una educación intercultural que garantice una educación más inclusiva y heterogénea, como heterogénea es la sociedad actual,que

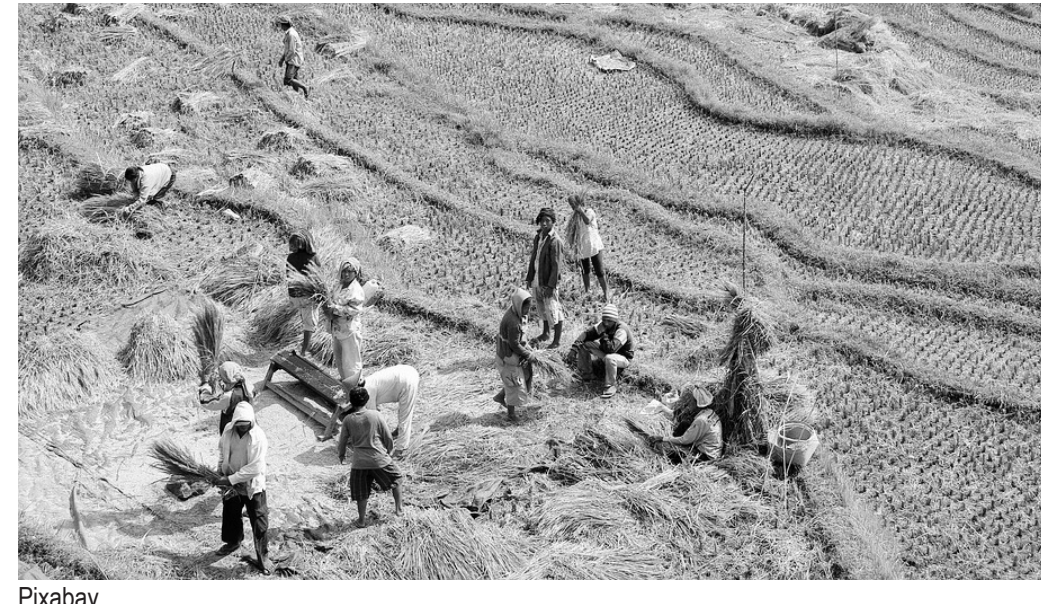

Pixabay

promueva una interacción armoniosa e igualitaria entre las persona diversas culturalmente. En este contexto uno de los organismos internacionales que promueve la educación intercultural es la UNESCO. En la Conferencia Internacional de Educación expresa que la Educación intercultural debe estar "destinada a todos los alumnos y ciudadanos, (...) promueve el respeto de la diversidad cultural, así como la comprensión y el enriquecimiento mutuo (UNESCO 1992. pág. 24).

Además en el informe final de esta Conferencia de Educación se recomendaron las siguientes metas para el desarrollo de la educación intercultural:

- La reducción de todas las formas de exclusión;

- El desarrollo de la integración y el logro escolar;

- La promoción del respeto de la diversidad cultural;

- El fomento del conocimiento de las culturas de otros;

- La promoción del entendimiento internacional

En este ámbito, la Educación Intercultural:

..debe abarcar el entorno pedagógico como un todo, al igual que otras dimensiones de los procesos educativos, tales como la vida escolar y la adopción de decisiones, la formación y capacitación de los docentes, los programas de estudio, las lenguas de instrucción, los métodos de enseñanza y las interacciones entre los educandos, así como los materiales pedagógicos. (UNESCO, 2006, p. 19).

Siguiendo los lineamientos de la UNESCO, implica que la cultura general, apertura otros conocimientos, lenguajes, competencia y valores 
que capacite a los educandos a desarrollar sus potencialidades culturales basándose en la diferencia, y promueve un espíritu de solidaridad y cooperación propiciando entre los individuos la instauración del diálogo y el respeto mutuo entre los diversos grupos sociales y culturales. Es decir la Educación Intercultural promueve una educación heterogénea, que responda y visibilice la diversidad cultural en el contexto educativo, para construir relaciones nuevas e igualitarias entre los diferentes grupos socioculturales lo que supone empoderar a aquellos que fueron considerados inferiores. La Educación intercultural plantea que las diferencias culturales sea una fortaleza en la educación y no una debilidad, además debe estar dirigida a todos los estudiantes y no a los grupos minoritarios diferentes culturalmente.

Considerando los principios planteados por la UNESCO, la Educación Intercultural, propicia condiciones necesarias y un clima favorable para el desarrollo de la Educación rural, respondiendo a las necesidades de cada población, además la educación intercultural respeta la diversidad cultural, la realidad social y económica, crea un clima de confianza, respeto, tolerancia y aceptación de la diversidad cultural en el contexto educativo y social, sin embargo lo más importante es que permite conocer, reconocer y aceptar su verdadera identidad cultural y el derecho a la diferente, conservando y valorando sus raíces culturales, que le sirvan como un nexo para integrarse a la sociedad mayoritaria, enriqueciéndose mutuamente.

\section{BIBLIOGRAFIA}

- FAO - UNESCO (2004). Educación para la población rural en Brasil, Chile, Colombia, Honduras, México, Paraguay y Perú. Proyecto FAO-UNESCO - DGCS/ ITALIA -CIDE -REDUC, Roma-Italia. Consultado 14 de mayo de 2013 de ftp://ftp.fao.org/docrep/ fao/009/y5517s/y5517s.pdf

- Aguado, T. (2005). La educación intercultural en la práctica escolar. Investigación en el ámbito español. Revista de Educación. Vol. XXI. núm. 7

- INDH (2010). Informe anual 2010, Chile. Consultado el 20 de enero del 2016 http://www.indh.cl/ wp-content/uploads/2010/12/Informe_Final_ Corregido1.pdf

- ONU (1960). Convención relativa a la lucha contra las discriminaciones en la esfera de la enseñanza, París. Consultado el 18 de mayo del 2013 dehttp://portal. unesco.org/es/ev.php-URL_ID $=12949 \& U R L_{-}$ DO=DO_TOPIC\&URL_SECTION=201.html

- UNESCO (1974). Actas de Conferencia General, París. Consultado el 18 de mayo del 2013 de http:/ / unesdoc.unesco.org/images/0011/001140/114040S. pdf

- UNESCO (1985). Actas de la conferencia general, Sofía. Consultado el 4 de agosto del 2015 de http:/ / unesdoc.unesco.org/images/0006/000684/068427s. pdf

- Declaración mundial sobre educación para todos (1990), Jomtien - Tailandia. Consultado el 15 de enero del 2016 de http:/ / www.oei.es/efa2000jomtien.htm

- FAO-UNESCO (2004). Educación para el desarrollo rural: hacia nuevas respuestas de políticas. Consultado el 26 de abril del 2013 de http:/ / unesdoc. unesco.org/images/0013/001329/132994so.pdf

- UNESCO. (1992). Conferencia Internacional de Educación $\mathrm{N}^{\circ} 43$.

- UNESCO (2006) Directrices de la UNESCO sobre la educación intercultural, París. 